\title{
Small-Signal Modeling of Pulse-Width Modulated Switched-Mode Power Converters
}

R. D. MIDDLEBROOK, FELLOW, IEEE

Invited Paper
A power processing system is required to convert electrical energy from one voltage, current, or frequency to another with, ideally, 100-percent efficiency, together with adjustability of the conversion ratio. The allowable elements are switches, capacitors, and magnetic devices. Essential to the design is a knowledge of the transfer functions, and since the switching converter is nonlinear, a suitable modeling approach is needed. This paper discusses the state-space averaging method, which is an almost ideal compromise between accuracy and simplicity. The treatment here emphasizes the motivation and objectives of modeling by equivalent circuits based upon physical interpretation of state-space averaging, and is limited to pulse-width modulated dc-to-dc converters.

\section{INTRODUCTION}

Almost all electronic equipment contains a power supply, often with a regulated output. The so-called linear reg. ulator, originally predominant, has largely given way to the switching regulator having higher efficiency and smaller size and weight. Despite these obvious benefits, the switching power supply unfortunately also has some disadvantages, including conducted and radiated noise arising from the switching process.

Less apparent is the fact that a switching power supply presents an order of magnitude greater difficulty in design. Both linear and switching power supplies are feedback systems, and therefore can be characterized in terms of the familiar concepts of loop gain, bandwidth, and stability margins. Analysis, and hence design, of a linear regulator can be accomplished with use of standard linear circuit techniques incorporating small-signal linearized models of the nonlinear active devices. In contrast, the switching power supply feedback loop contains a modulator and a switching power stage that constitute a type of analog-todigital followed by a digital-to-analog conversion function,

Manuscript received September 21, 1987; revised January 6, 1988 This work was conducted in part under the Power Electronics Program supported by GTE Communication Systems Corporation, EG\&G Almond Instruments, and Rockwell Inc.

The author is with the Power Electronics Group, California Institute of Technology, Pasadena, CA 91125, USA.

IEEE Log Number $\mathbf{8 8 2 0 0 8 0 .}$ for which, at the outset, there was no model for analysis of the transfer functions.

Over more than 15 years, much has been achieved in modeling the nonlinear switching converter power stages and modulators, and also in application of the models to the design of improved, more cost-effective switching regulators.

Rather than attempt a review of the many approaches which, in the available space, would inevitably result in a superficial treatment of all of them, this paper instead will emphasize the motivations and objectives of modeling by one approach, state-space averaging, that represents an almost ideal tradeoff between accuracy and simplicity, which no doubt accounts for its widespread adoption.

In order to make the topic more easily accessible to nonspecialists, the scope will be even more limited: the bulk of this paper emphasizes the motivation and objectives of modeling by equivalent circuits, since these give physical insight into the qualitative behavior and are an essential prerequisite to proper use of the more abstract analytic and computational methods. Indeed, historically, the equivalent circuit approach preceded the generalization embodied in state-space averaging.

For the specialist reader, the paper offers a perspective on the equivalent circuit models, and their comparative properties for different operating modes, together with a summary of the formal state-space averaging method.

The work of the author, his colleagues, and students of the Power Electronics Group at the California Institute of Technology has been collected in [1]. Much of it concerns the subject of modeling, and illustrates the development of the viewpoint summarized here. Many other workers, some of whom are explicitly referenced, have also contributed to the subject of modeling, particularly in extensions of the state-space averaging method, as well as different approaches.

In Section II, a power processing system is compared and contrasted with a signal processing system. Not only are the objectives different, but so are the allowable elements from which they may be built. 
In Section III, a generalized dc-to-dc regulated power supply is used as a vehicle for introduction of the motivation and objectives of modeling. A switched-mode power stage and a "linear" power stage are both nonlinear systems, and the modeling objective is the same for each: to find a small-signal ac model whose element values are constant but functions of the large-signal dc operating point.

In Section IV, canonical models are established in a qualitative manner for pulse-width-modulated switched-mode converters in various modes of operation. A canonical model is merely an equivalent circuit that has a fixed configuration, regardless of the type of converter it represents, although its element values are different for different converters.

A qualitative comparison of the properties of the various operating modes for different converters is made in Section $V$. This is the purpose of modeling, so that the designer is aware of the nature of the transfer functions to be dealt with in design of the regulator feedback loop.

The detailed foundation of the modeling described in the earlier sections is state-space averaging, reviewed in Section VI. Equivalent circuit models are one of several useful forms of the results; others are analytic expressions for frequency responses, and numerical outputs of individual transfer functions.

Conclusions are drawn in Section VII. The state-space averaging method does not accommodate another important class of power processing systems, namely resonant converters, and methods for their analysis are not mentioned in this paper; nor are modeling methods for dc-toac inverters.

\section{Design Constraints and Objectives of Power PROCESSING SYSTEMS}

Power processing systems, contrasted with signal processing systems, are required to process power with minimum power loss, rather than to process information with minimum power consumption. The function of electrical power processing systems is to convert electrical energy from one voltage, current, or frequency to another. This function is to be achieved, ideally, with 100-percent efficiency. In most applications, including those of greatest technical interest, the conversion is to be accomplished adjustably; this is so that a regulated output can be obtained by closing a feedback loop that causes the conversion ratio to be automatically adjusted.

The term power processing suggests an intentional contrast with signal processing, a function much more familiar to most modern electronics engineers. As indicated in Fig. $1(a)$, a signal processing system is concerned with performing operations upon the information content of an input: typical functions are analog amplification, digital recoding, analog-to-digital and digital-to-analog conversions. To perform these functions, electrical power is required, usually in $\mathrm{dc}$ form and in an amount to be minimized.

In a power processing system, as indicated in Fig. 1(b), the "power" and "information" inputs are interchanged, and the concern is with performing operations upon the power input according to the functions specified by the information input. Typical functions are dc-to-dc conversion, dc-to-ac inversion, ac-to-dc rectification, and ac-to-ac cycloinversion.

The distinctions between signal and power processing
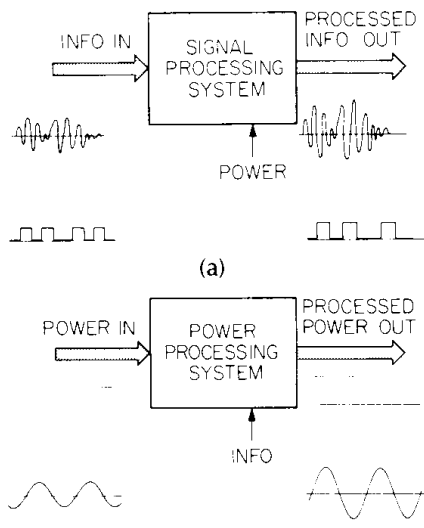

(b)

Fig. 1. A signal processing system (a), contrasted with a power processing system (b).

systems extend down to the most basic level of design: the selection of elements available for use by the engineer. In somewhat oversimplified summary, these are resistors, inductors, capacitors, and active devices that can be used either in linear (analog) mode or in switching (digital) mode. All these elements are available to the signal processing integrated-circuit designer-except the inductors. Magnetic elements are shunned because they are bulky, heavy, expensive, and do not (yet) fit conveniently on to semiconductor chips. Indeed, it is convenient to develop, when necessary, quite complicated circuitry to simulate inductor properties. Of course, what is being simulated is $L d i / d t$, the signal processing function, not $\frac{1}{2} L i^{2}$, the energy storage function needed for power processing.

In contrast, the power processing designer has at his disposal a different array of elements: resistors are now anathema because they are lossy and do not meet the efficiency requirement. For the same reason, linear operation of active devices must be excluded, which leaves only inductors, capacitors, and switches as allowable elements. Of course, transformers and a wide variety of more complex magnetically coupled structures are also included, and, indeed, exploitation of these possibilities is one of the greatest current challenges in the power electronics field [2].

It is seen that the different set of available elements requires the power processing designer to develop a quite different way of thinking from that of the signal processing designer. One aspect of this is the recognition of a conflict unique to the power processing field: minimization of the parasitic resistances of the magnetics, capacitors, and switches, desirable in the interests of efficiency, leads also to undesirable minimum damping of the inherent $L C$ filters, with consequent increased difficulties in design of the feedback loop and in the dynamic range. As usual, proper definition of the problem constitutes a large part of its solution: what is needed is lossless damping, of which more will be said later.

One means of converting one voltage/current level to another is the conventional transformer. Such a practical device can approach very closely the ideal 100-percent efficiency, especially in large sizes. However, from the point of view of power processing, it suffers from two severe limitations: it does not change frequency, and it does not operate at dc. Although its capabilities are limited, the physical 
transformer nevertheless suggests a characterization of the general power conversion functions, namely, conversion of one voltage/current/frequency combination to another, in which dc is included as zero-frequency ac.

In turn, this generalization suggests a useful representation, or model, of the power conversion function: the circuit symbol shown in Fig. 2(a), an adjustable-ratio ideal
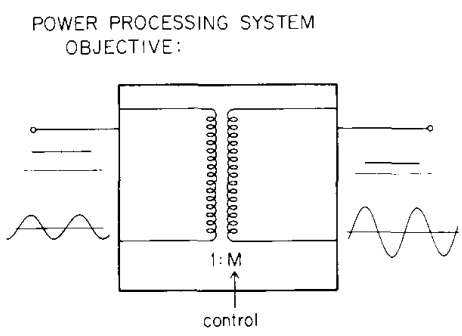

Adjustoble - ratio ideal transformer:

$d c-10-d c, d c-10-a c, a c-10-d c, a c-10-a c$

(a)

REALIZATION:

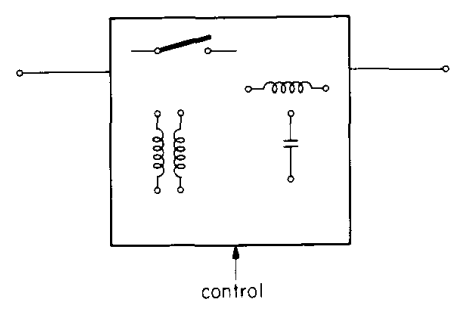

Switched-mode conversion: approaches $100 \%$ efficiency

(b)

Fig. 2. (a) Objective of a power processing system; (b) its realization by an optimal arrangement of the allowable elements. transformer. The most general objective of the power processing design may then be crystallized thus: How to find an optimum arrangement of the allowed circuit elements, as suggested in Fig. 2(b), in order to achieve the conversion functions shown in Fig. 2(a)? Part of the answer lies in knowledge of the small-signal ac properties of the various possible configurations.

\section{Motivation and Objectives of Modeling}

Of the four processing functions designated in Fig. 1(b), the modeling approach for only one, dc-to-dc conversion, will be discussed in this paper. The essence of such a dc regulated power supply is shown in Fig. 3. For illustration, the switching converter is a simple buck (voltage step-down) circuit; any other converter, such as the basic boost (voltage step-up) or flyback (step-up or step-down), shown in Fig. 4, could be substituted. In all of these, the transistor and diode operate as a single-pole switch.

In Fig. 3, the unregulated line input at voltage $v_{g}$ passes through a switching converter to the output of voltage $v$, from which an output current $i$ is drawn requiring a line input current $i_{g}$. The transistor switch is controlled by a duty ratio (digital) signal $d$ from the output of a modulator, which is usually a simple comparator whose inputs are a clock sawtooth or triangular waveform and an (analog) control signal. In the waveforms shown here, the modulator turns on the transistor switch uniformly at the beginning of each clock cycle, and turns it off when the control signal overtakes the ramp. If the control signal varies, so does the duty ratio. This constant frequency, pulse-width modulated (pwm) transistor switch, together with the power diode, present a square voltage waveform to the $L C$ filter, which in turn delivers the average component of this waveform to the output with small residual switching frequency ripple.

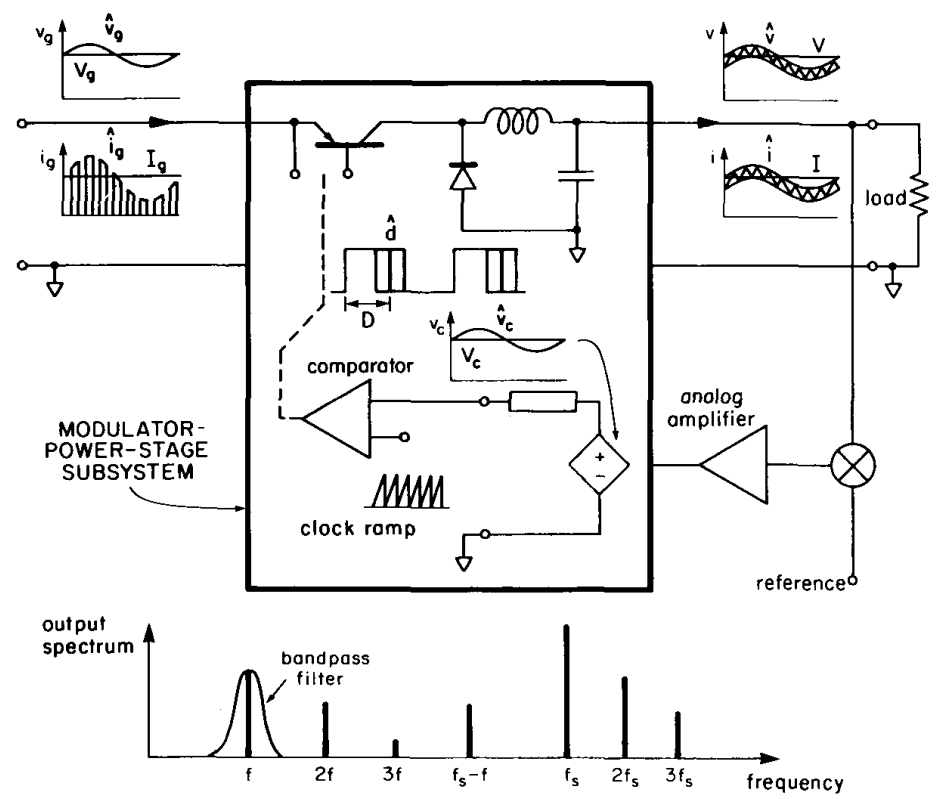

Fig. 3. A dc-to-dc switching regulator incorporating a three-port duty ratio programmed modulator-power-stage subsystem whose transfer functions are defined in terms of ratios of small-signal ac quantities (hats) superimposed upon large-signal dc quantities (capitals). The spectrum of the output signal contains the switching frequency, the control frequency, their respective harmonics, and sidebands. 

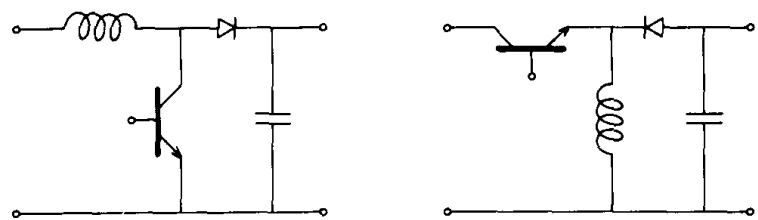

Fig. 4. Left: basic boost converter. Right: basic flyback (buck-boost) converter.

The regulator feedback loop is closed via the sensed converter output which is compared with the reference, and the (analog) amplifier error signal constitutes the control signal to the modulator. The error amplifier output is represented by a Thevenin equivalent voltage source $v_{c}$ and series impedance (diamonds are used to distinguish dependent from independent sources represented by circles).

The ultimate analysis objective is to find, as functions of frequency, the loop gain and hence the closed-loop properties of the regulator. The essential prerequisite is to find the transfer functions of the three-port subsystem inside the large box in Fig. 3, designated the modulator-powerstage subsystem. The most important of these are the control-to-output and line-to-output transfer functions and, secondarily, the converter output and input impedances.

If the subsystem inside the boxes in Fig. 3 were linear, the control input, represented by the sum of dc and ac components $v_{c}=V_{c}+v_{c}$, would lead to a corresponding converter output $v=V+\hat{v}$ in which the ac component $\hat{v}$ would be at the same frequency as $\hat{v}_{c}$ and proportional to it, so that the control-to-output ac transfer function would be $\hat{v} / \hat{v}_{c}$ independent of the amplitude of $\hat{v}_{c}$. The same would be true of any transfer function.

However, the subsystem inside the box is not linear; nevertheless, the objective is still to find the transfer functions, albeit subject to some approximation. There will be a price to pay for the resulting simplification, namely, restrictions on the validity of the result.

It is to be noted that this problem is not peculiar to the switching converter subsystem. If the subsystem is a socalled linear regulator, exactly the same problem exists [3]. A transistor operated in the active region is a nonlinear device, and the solution to the problem lies in finding a model that represents the small-signal ac properties at a given large-signal operating point. The resulting model, for each transistor, contains elements whose values may be functions of operating point, but are otherwise independent of the ac signal amplitude as long as it is small enough. There are many such models, known as the tee, hybrid- $\pi$, $y$-parameter, etc. In the tee model, for example, the value of the emitter resistance is inversely proportional to the emitter dc operating current, but is taken to be constant with respect to sufficiently small ac signals.

It is simple, yet significant, to say that the sole purpose of an equivalent circuit model is to give the right answer for the transfer functions. In the case of the switching converter subsystem in Fig. 3, the required transfer functions are those of the three-port subsystem, and so an equivalent circuit model representing the whole box, not just one transistor, is required. The objective, however, is the same: to find a linear equivalent circuit that represents the properties of terminal small-signal ac variations, in which the nonlinearities are relegated to the variation of the element values with large-signal dc operating point. The operating point is defined by the dc values $V_{g}, l, V_{c}$ etc.

In addition to the nonlinearity that causes harmonics of the control signal frequency $f$ to occur in the output, a switching converter presents an additional problem: the presence in the output of the switching frequency $f_{s}$ and its harmonics, and also sidebands $f_{s}-f$, etc. The spectrum of the output voltage is also illustrated in Fig. 3. Fortunately, this problem is not as severe as it may seem, because the basic requirement for low output switching ripple requires that the low-pass filter corner frequency be well below the switching frequency $f_{s}$ by at least a decade. This then highly accentuates the component of the output at the control fundamental frequency $f$, and de-emphasizes all the other frequencies.

Thus, the linearized part of the transfer properties consists of the output signal (magnitude and phase) at the same frequency as the control signal $\hat{v}_{c}$. Hence, as before, a control-to-output transfer function for the linearized system can be defined as $v / \hat{v}_{c}$, and similarly for the other transfer functions.

Suitable models from which the transfer functions can be found are discussed in the next section.

\section{Small-Signal Canonical Models for DC-to-DC CONVERTERS}

With the motivation and objectives of the model of the modulator-power-stage subsystem in Fig. 3 now clearly delineated, establishment of the model itself is but a final short step, at least qualitatively.

As already seen from the description of the operation, the control-to-output transfer function $v / v_{c}$ can be considered made up of two parts, the modulator transfer function $d / v_{c}$ and the power stage transfer function $v / d$. The first part is very simple, being merely a proportionality factor $1 / V_{m}$, where $V_{m}$ is the height of the clock ramp. This follows because the duty ratio $d$ is zero when the control voltage $v_{c}$ is zero, is unity when the control voltage is $V_{m}$ and is linear in between if the ramp is linear. The ramp need not belinear, in which case $V_{m}$ is the equivalent height of a ramp having the same slope as the actual ramp at a given operating point.

The model of the power stage can be established by introduction of components that represent the three essential features of any dc-to-dc pwm switching converter, all of which have already been mentioned.

The first essential feature is the dc-to-dc conversion property itself, already represented by the generalized ideal transformer symbol of Fig. 2(a), characterized by the conversion ratio $M$.

The second essential feature is the presence of a low-pass, essentially lossless filter, since in any dc-to-dc converter the switching frequency is to be heavily attenuated in the output. This feature may be represented directly by a low-pass LC filter.

The third essential feature is adjustability of the conversion ratio, which is implicit in the dependence of $M$ upon the duty ratio $d$. This is inconvenient with respect to application of the model, because when $d$ is varied according to the control voltage $v_{c}$, then $M=M(d)=M(D+d)$ also varies and makes the transformer a nonlinear element. In accordance with the procedure for linearizing a nonlinear 
element described in Section III, the transformer is characterized by a conversion ratio fixed at the value $M(D)$ corresponding to a given operating-point duty ratio $D$, and the $d$ variation component is separated out into two "modulation" generators proportional to $d$, where the proportionality factors are functions of large-signal operating point. This process, introduced in [4] and described in detail in [5] and [6], involves the usual small-signal restriction in order to eliminate the nonlinearity. Both a current and a voltage modulation generator are necessary, because a variation of the conversion ratio affects both current and voltage.

The three model components representing the three essential features are assembled in Fig. 5 [4]. A further

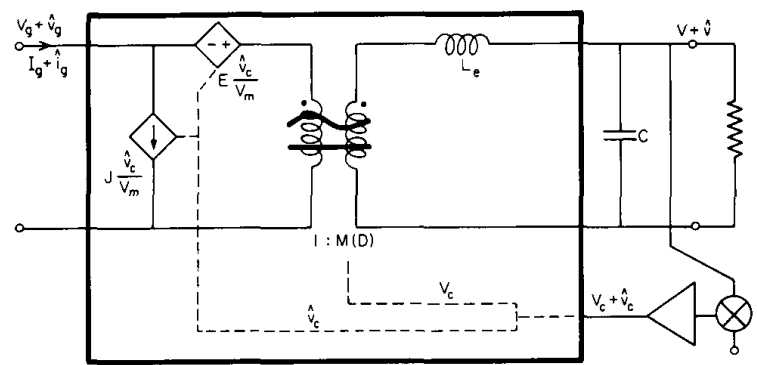

Fig. 5. Canonical model for pwm converter in duty ratio programmed continuous conduction mode. This model contains the three essential features: dc-to-dc conversion transformer, low-pass filter, and modulation generators.

reduction is also included: the simple proportionality of the modulator transfer function has been inserted into the modulation generator coefficients, which are now driven by $\hat{v}_{c}$ instead of by $d$. The total control voltage $v_{c}=v_{c}+$ $\hat{V}_{c}$ is thus separated into two parts, and the dc component $V_{c}$ determines the dc (operating point) duty ratio $D=V_{c}$ l $V_{m}$ of which the transformer conversion ratio is a function. This function $M(D)$ is different in different converters, and may be nonlinear. It may be noted that the transformer symbol is modified by imposition of a straight and a wavy line, as a reminder that it represents a generalized conversion function that extends to zero frequency.

Qualitatively, the model in Fig. 5 represents the transfer function properties of any dc-to-dc pwm switching converter, regardless of its actual configuration (topology), and is therefore designated a canonical model. The values of the elements, and their dependences upon operating point do, however, depend on the converter type and, more drastically, on the converter mode of operation.

\section{A. Canonical Model for Duty-Ratio Programmed Continuous Conduction Mode}

In the description of Fig. 3, it was mentioned that the transistor and diode operate as a single-pole switch. Further examination reveals, however, that the single-pole switch can operate in either a two-position or a three-position mode, per cycle of the switching frequency.

When the transistor is $\mathrm{ON}$, the filter inductor is connected to the line input, the diode is OFF, and the inductor current ramps up (essentially linearly if the filter corner frequency is much lower than the switching frequency, which is always the case in pwm converters). When the transistor is turned OFF by the modulator, the inductor current commutates to the diode, which therefore at the same time is automatically turned $\mathrm{ON}$. The inductor current thereupon ramps down. If the inductor current has not reached zero at the end of the switching period when the modulator again turns the transistor $\mathrm{ON}$, a continuous conduction mode $(\mathrm{ccm})$ of the inductor current exists in which the transistor and diode are alternately ON and OFF and operate as a twoposition single-pole switch. The converter then constitutes a two-switched network.

In ccm, the canonical model of Fig. 5 [7] is useful directly because the transformer conversion ratio is a function only of duty ratio $D$. Thus, the control voltage $V_{c}$ uniquely determines the duty ratio $D$, which can be described as duty ratio programming, and in turn the duty ratio $D$ uniquely determines the converter output voltage $V$ (for a given line voltage $V_{g}$ ) so that, overall, the converter can be described as voltage programmed in that the control voltage uniquely determines the output voltage.

Quantitatively, in ccm, the specific dependences of the canonical model element values upon converter type are as follows. In the simple buck converter chosen for illustration in Fig. 3, the filter in the model directly represents the physical filter; however, in some converters, notably the boost and the buck-boost (also known as the flyback) the inductance in the model has an effective value related to the physical inductor value, but is also a function of operating point.

In the buck converter, both modulation generators, while dependent upon operating point, are independent of frequency; however, again notably in the boost and flyback converters, the voltage generator is also a function of frequency in the form of a right half-plane (rhp) zero, which is also a function of operating point.

\section{B. Canonical Model for Duty-Ratio Programmed Discontinuous Conduction Mode}

As described above, the continuous conduction mode exists if, when ramping down during the transistor OFFtime, the inductor current does not reach zero. Conversely, if the inductor current does reach zero before the modulator turns the transistor $\mathrm{ON}$ again, the diode current tries to reverse but cannot, and the diode thereupon turns OFF. Following the transistor ON, diode OFF and transistor OFF, diode ON intervals, there is therefore a third interval during which both transistor and diode are OFF and the inductor current remains at zero. The transistor and diode therefore operate as a three-position single-pole switch per cycle of the switching frequency, and the converter then constitutes a three-switched network described as the discontinuous conduction mode $(\mathrm{dcm})$ of the inductor current. Transition from continuous to discontinuous mode occurs when the converter load current falls below some minimum value, usually designed to be about 10 percent of the maximum load current.

In dcm, the canonical model of Fig. 5 is not useful directly because the element values become strong functions of the load current. In particular, the transformer conversion ratio becomes a function not only of the duty ratio $D$, but also of a parameter $K=2 f_{s} L / R$, so that $M=M(D, K)$, where $L$ is the filter inductance value and $R$ is an operating point parameter defined as the ratio of the dc load voltage to the dc load current. For a resistive load, $R$ is the same as the load 
resistance. Because of the dependence of $M$ upon $R$, it is no longer convenient to model the conversion ratio by a transformer since the current and voltage transfer properties become unequal. For this and other reasons it is more useful to represent the discontinuous conduction mode by a $y$-parameter model, as shown in Fig. 6.

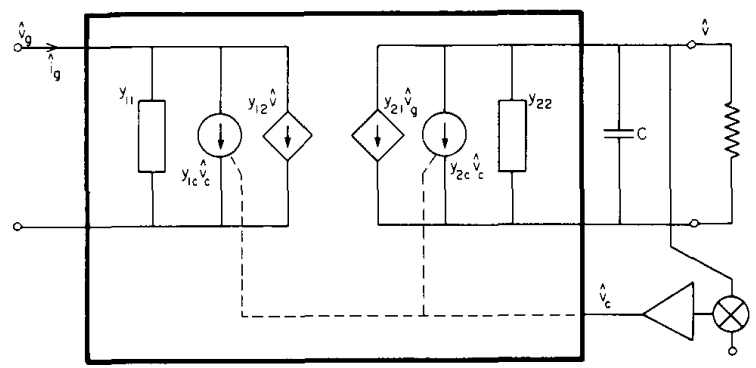

Fig. 6. Canonical model for pwm converter in duty ratio programmed discontinuous conduction mode, and also in current-programmed continuous conduction mode. The output current is only approximately programmed by the control voltage, since the resistive component of the output admittance is comparable to the load resistance.

The model of Fig. 6 [8] is again a canonical model, because it represents qualitatively the properties of any converter regardless of its detailed topology. The two dependent generators $y_{21}$ and $y_{12}$ are unequal, so cannot be replaced by a transformer, confirming that the network is nonreciprocal. The two modulation generators remain, albeit in a different format as $y_{2 c}$ and $y_{1 c}$. The inductance, however, is notably absent explicitly; this is because the inductor current, in discontinuous conduction, starts and ends each switching cycle at zero, and therefore drops out as a state variable of the system. It is, however, implicitly present via the dependence of some of the element values upon the parameter $K$. So is the switching frequency implicitly present through the same parameter $K$, whereas it was absent altogether from the canonical model for continuous conduction mode, other than as an upper frequency limit upon the validity of the model as a whole.

Both $\mathrm{ccm}$ and $\mathrm{dcm}$ modes of operation are duty ratio programmed, in that the duty ratio is uniquely determined by the modulator control voltage. For the $\mathrm{ccm}$, the canonical model of Fig. 5 shows that the transformer conversion ratio is solely a function of duty ratio and so, as already mentioned, the output voltage is also programmed by the control, because the output resistance is contributed only by parasitic losses.

On the other hand, in $\mathrm{dcm}$, the resistive component of the output admittance $y_{22}$ in the canonical model of Fig. 6 is not small, but in fact is comparable to the resistive component of the load. Consequently, in this mode the converter cannot be said to be either voltage or current programmed.

\section{Canonical Model for Current-Programmed Continuous} Conduction Mode

Any duty ratio programmed pwm switching converter topology having a modulator as shown in Fig. 3 may operate in either the continuous or discontinuous inductor current mode. Although such configurations are still considered "standard," a different modulator connection, which is not duty ratio programmed, has rapidly gained acceptance over the last ten years [9].

Variously known as "current," "current-mode," or "current-reference" programmed, this configuration is shown in Fig. 7. The principal difference is that the fixed clock ramp

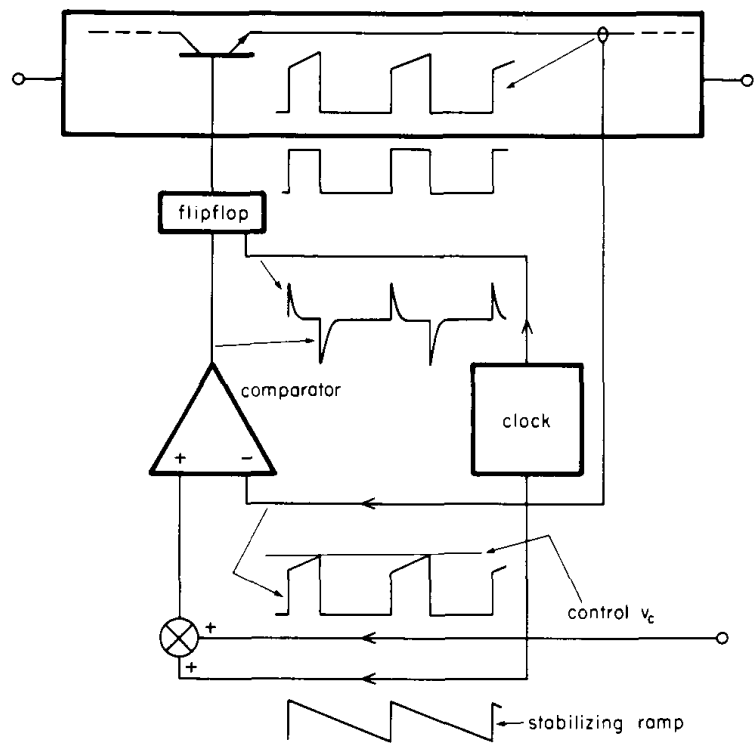

Fig. 7. A current-programmed modulator-power-stage subsystem incorporating a minor current feedback loop, and a stabilizing ramp derived from the clock.

input to the modulator (comparator) is replaced by a signal proportional to the ramp waveform of the power stage switch current, which is also the current through an inductor regardless of the power stage topology. The clock, as before, initiates the switch ON-time uniformly at the switching frequency $f_{s}$. However, the switch ON-time is now terminated when the ramping-up inductor current reaches a value proportional to the control signal $v_{c}$. Clearly, a physical "minor" current feedback loop exists within the modulator-power-stage subsystem, inside the "major" voltage feedback loop of the regulator of Fig. 3 .

Instability of the minor loop occurs for a duty ratio 0.5 or greater, unless a stabilizing ramp of suitable slope is applied to the modulator input [9], [10]. This stabilizing ramp is easily derived from the clock, as indicated in Fig. 7.

The immediate consequence of the minor feedback loop is that its sensed "output," the switch/inductor current, tends to follow its "input," the control signal $v_{c}$; in other words, this current is programmed. As a first approximation, therefore, the entire subsystem in the box of Fig. 3 could be modeled (at least as far as the control-to-output transfer function is concerned) by a current generator across the output, dependent upon the control signal.

It turns out, however, that this model is rather too simple. One approach to a more detailed treatment [10] is to start with the canonical model for the duty ratio programmed power stage, and to add to it the model of the modulator obtained from analysis of the comparator input waveforms of Fig. 7. An example is shown in Fig. 8, for the boost converter in continuous conduction mode. 


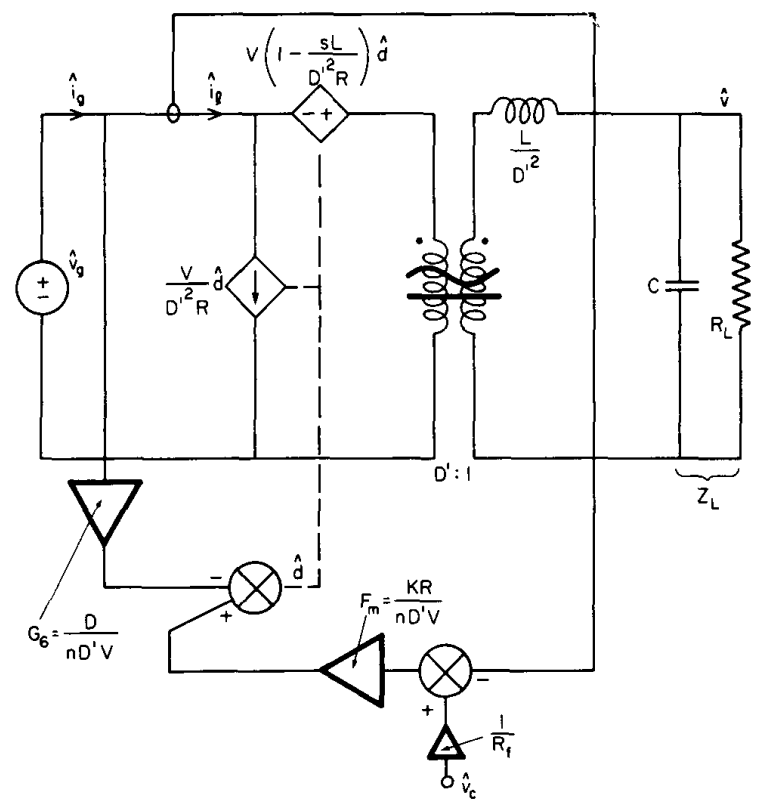

Fig. 8. Basic model of the current-programmed boost converter, showing the current loop via $F_{m}$ closed around the canonical model for the duty ratio programmed mode.

The modulator transfer function $d / \hat{v}_{c}$ is no longer a simple proportionality, since the modulator now has two additional inputs, the inductor current and the line voltage. The line voltage appears because in the boost converter, during the switch ON-time, the line voltage appears across the inductor and determines the slope of its current ramp. In the buck converter, during the switch ON-time, the difference between the line and output voltages appears across the inductor, and consequently the output voltage is yet another input to the modulator.

The model of Fig. 8, or the corresponding one for another converter, can be analyzed for the loop gain of the minor current feedback loop. In all cases it is found to be quite low [10], in the range 1 to 10 at low frequencies and, moreover, declines below unity at a crossover frequency $f_{\mathrm{c}}$ which is between one-sixth and two-thirds of the switching frequency.

Although the model of Fig. 8 could be used directly in the analysis and design of the complete regulator, it is simpler first to reduce this model to a canonical form [10], [11]. In this way, the current feeback is absorbed into the canonical model, which in turn can be used as a component in the analysis of the regulator major voltage feedback loop in the usua! manner. Also, such a canonical model of the subsystem box of Fig. 3 exposes the properties of the power stage independently of the major voltage loop and, in particular, shows how successfully the current programming causes the switch/inductor current to follow the control signal-not very successfully, it turns out.

As already mentioned, a first-order model of the currentprogrammed power stage consists merely of an output current generator dependent upon the control signal. It is reasonable, therefore, to choose a more general model that includes such a generator as one of its elements. The obvious choice is the $y$-parameter mode!.

Hence, the $y$-parameter model of Fig. 6 is suitable not only to represent the duty ratio programmed subsystem of Fig. 3 in discontinuous conduction mode, but also the currentprogrammed subsystem in continuous conduction mode. The performance similarity between the two modes even extends quantitatively: the resistive component of $y_{22}$ in current-programmed mode is also on the order of the output operating point parameter (the load resistance in the case of resistive load), as it is for $\mathrm{dcm}$, which means that the output is not a stiff current source, which in turn means that the "current programming" is not particularly effective.

There are two reasons why the current feedback loop, now buried inside the $y$-parameter model, does not lead to a stiff current source at the power stage output. One reason, already mentioned, is that the current loop gain is quite low, so that the sensed current does not track the control signal very closely. The other reason is that the current being sensed, the switch/inductor current, is not necessarily the power stage output current that is being modeled by the $y$-parameters. In the buck converter, the inductor current is the output current, but in the boost and flyback, for example, it is not (because the inductor is not in series with the output), and so there is an additional transfer function between the inductor current and the output current that also leads to a less stiff current source in the model of the output properties.

It may be concluded that the power stage and modulator subsystem of Fig. 7 hardly deserves the name "current-programmed," since the current programming is no more effective than in the case of the duty ratio programmed discontinuous conduction mode. In other words, both subsystems are about equally well (or equally poorly) current programmed, at least in comparison with the stiff voltage programming that occurs in the duty ratio programmed continuous conduction mode of operation. It is, however, too late to suggest any change in the established descriptions.

The canonical models can easily be extended to represent the properties of many derived converter topologies, such as the push-pull, forward, and other transformer-coupled versions [12].

\section{Comparison of Properties of Different Modes of OPERATION}

The purpose of the canonical models established in the previous section is to permit easy analysis of the various transfer functions, and to provide insight into the qualitative behavior. This is an intermediate step towards determination of the regulator major loop gain and, hence, the closed-loop properties of the regulator that must meet design specifications.

In this section, a qualitative comparison is made of the properties represented by the three canonical models, with respect to only the most important of the transfer functions, the control-to-output.

The three models discussed are for duty ratio programmed continuous and discontinuous inductor current modes of operation, and for current-programmed continuous mode of operation. It has already been seen that the second and third modes have quite similar properties, both represented by the $y$-parameter model of Fig. 6 . There is a fourth mode, current-programmed discontinuous inductor current, but its properties are similar to those of the second and third modes, and will not be discussed. 
The control-to-output transfer function $\hat{v} / \hat{v}_{c}$ for duty ratio programmed $\mathrm{ccm}$ can be found from the canonical model of Fig. 5. For zero line source impedance, the current modulation generator is shorted out, and the voltage modulation generator thus appears across the conversion transformer primary. The function $\hat{v} / \hat{v}_{c}$ therefore consists of three factors: the coefficient of the voltage modulation generator, the conversion ratio $M$, and the filter voltage transfer function.

In the case of a buck converter power stage, the voltage modulation generator coefficient is independent of frequency and hence the frequency response of the controlto-output transfer function is simply that of the filter. Also for the buck, the filter effective inductance $L_{e}$ is equal to the physical inductor value $L$, and so the filter characteristic is constant, independent of operating point.

Typically, the $L C$ filter has a $Q$ of at least 0.5 , and is therefore underdamped and has a transfer function characterized by a complex pole pair. The resultant peaking in the response is undesirable for several reasons: the accompanying steep phase lag in the control-to-output transfer function causes stability difficulties in the regulator major voltage loop gain, and also the peaking restricts the available dynamic range of ac load current components.

These properties are the core of the design conflict already alluded to in Section II: the lower the losses, the higher the $Q$, and the less desirable the dynamic response. Hence, higher efficiency involves greater dynamic design difficulty [13]. One resolution of this conflict can be achieved by placing a dc-blocked damping resistance across the filter capacitor; this is an example of lossless damping [14].

Both the boost and flyback power stages, in continuous conduction mode, exhibit even greater design difficulties. The voltage modulation generator in the canonical model of Fig. 5 contains an rhp zero, so the control-to-output transfer function contains the rhp zero in addition to the two (complex) poles of the filter. The $270^{\circ}$ asymptotic phase lag severely restricts the allowable bandwidth of the regulator voltage loop gain, since the rhp zero can lie considerably below the switching frequency. Worse, not only does the rhp zero change with operating point, but so do the filter poles, because the effective inductance itself becomes a function of the duty ratio.

In duty ratio programmed $\mathrm{dcm}$, the $y$-parameter canonical model of Fig. 6 shows that the control-to-output transfer function is considerably simpler, for all basic power stage topologies. The only model elements needed to solve for $\hat{v} / \hat{v}_{c}$ are $y_{2 c}$ and $y_{22} ;$ although both depend on operating point, neither is a function of frequency, and so $y_{22}$ is a resistance $R_{22}$ whose value, as al ready mentioned, is comparable with the converter output operating point parameter $R \equiv$ $V / I$. Hence, for a resistive load $R_{L}, \hat{v} / \hat{v}_{c}$ is a single-pole function in which the pole is determined by $R_{22} \| R_{L}$ in parallel with the output capacitance $C$.

Although the single-pole response is much easier to handle in the regulator loop design than is the high- $Q$ doublepole response, there are disadvantages of the discontinuous compared to the continuous conduction mode. Since the inductor current starts and ends each switching cycle at zero, its rms value is always higher in relation to its $\mathrm{dc}$ value than in continuous conduction, with consequent higher losses. Also, there is higher relative noise. For these reasons, use of discontinuous conduction mode is usually limited to converters for relatively low power ratings.
Converters operating in current-programmed continuous mode are also represented by the $y$-parameter canonical model of Fig. 6, although the expressions for the element values are somewhat more complicated. In particular, all six elements contain a pole at the current feedback loop crossover frequency $f_{c}$, which is a manifestation of the usual result that the effects of feed back disappear above the loop gain crossover frequency.

The pole at $f_{\mathrm{c}}$ in the output admittance $y_{22}$ can be ignored, because at this frequency the output capacitance $C$ already dominates the total load on the $y_{21}$ and $y_{2 c}$ generators. The remaining resistive component $R_{22}$ of $y_{22}$, as already mentioned, is comparable to the operating point parameter $R$ $=V / I$ and hence, for a resistive load $R_{L}$, as in the case of the discontinuous conduction mode, there is a pole in the control-to-output transfer function $\hat{v} / \hat{v}_{c}$ determined by $R_{22} \| R_{L}$ in parallel with the output capacitance $C$.

In current-programmed mode, however, there is a second pole in $\hat{v} / \hat{v}_{C}$ that comes directly from the pole $f_{C}$ in the $y_{2 c}$ generator of Fig. 6 . Hence, the control-to-output transfer function in current-programmed mode is a two-pole function, although one pole is dominant (that due to $C$ ), and the other pole is at a much higher frequency ( $f_{c}$, between onesixth and two-thirds of the switching frequency) [10]. In other words, the two poles are characterized by a low Qfactor, much less than 0.5 and therefore heavily damped. Current programming, consequently, introduces the desirable effect of lossless damping, lossless because the damping results from the minor current feedback loop, and not from physical (lossy) resistance. This so-called "single-pole response" is commonly quoted as one of the principal benefits of current programming; however, "dominant-pole response" would be a more appropriate term, since the additional phase lag from the second pole can have a significant influence on the phase margin of the regulator major voltage feedback loop.

A comparison of the most important features of the canonical models for the three operation modes that have been discussed is displayed in Table 1. The "approximately current programmed" description refers to the fact, already discussed, that the output resistance is comparable to the value of the (resistive) load, and is therefore relatively high compared to that of a stiff voltage source, although not high enough to constitute a stiff current source. The only feature not so far mentioned is that the rhp zero in the boost or flyback converter is present in the current programmed as well as duty ratio programmed continuous conduction modes. This is because the duty ratio programmed model is embedded in the current programmed model, as seen in the example of the boost converter in Fig. 8, and the current minor feedback loop does not affect any zeros in the control-to-output transfer function.

It is seen from Table 1 that the dynamic properties of the current programmed mode of operation are in between those of the duty ratio programmed continuous and discontinuous modes. Moreover, the current programmed mode combines the advantages of the other two modes, the continuous conduction operation together with the dominant pole response, for the price only of retention of the rhp zero in the boost and flyback converters.

There are two other significant advantages of the current programmed mode of operation, not directly related to the small-signal response: implementation of the current sense inherently provides almost all the components necessary 
Table 1 Relative Properties of the Control-to-Output Transfer Function of pwm Converters in Three Modes of Operation

\begin{tabular}{|c|c|c|c|}
\hline \multirow[b]{2}{*}{ Operation Mode } & \multirow[b]{2}{*}{ Output } & \multicolumn{2}{|c|}{$\begin{array}{l}\text { Control-to-Output } \\
\text { Transfer Function }\end{array}$} \\
\hline & & Poles & $\begin{array}{l}\text { rhp zero in } \\
\text { Boost and } \\
\text { Flyback }\end{array}$ \\
\hline $\begin{array}{l}\text { Duty ratio programmed } \\
\text { continuous inductor } \\
\text { current mode }\end{array}$ & voltage programmed & $\begin{array}{l}\text { complex pair } \\
\text { (high Q) }\end{array}$ & yes \\
\hline $\begin{array}{l}\text { Duty ratio programmed } \\
\text { discontinuous inductor } \\
\text { current mode }\end{array}$ & $\begin{array}{l}\text { approx. current } \\
\text { programmed }\end{array}$ & single & no \\
\hline $\begin{array}{l}\text { Current programmed } \\
\text { continuous inductor } \\
\text { current mode }\end{array}$ & $\begin{array}{l}\text { approx. current } \\
\text { programmed }\end{array}$ & $\begin{array}{l}\text { real pair, one } \\
\text { dominant } \\
\text { (low } Q \text { ) }\end{array}$ & yes \\
\hline
\end{tabular}

for a cycle-by-cycle current limit on the power switch, and the high output resistance permits paralleling of power stages inside the same voltage regulator loop, greatly enhancing the designability of modular power supplies. All these features have led to current programming becoming the preferred mode of operation for most pwm switching converters.

\section{The State-Space Averaging Method}

The description of converter small-signal models given in the previous section is a circuit-oriented approach, aimed at equivalent-circuit results having elements that retain direct physical interpretation. Indeed, this is the way in which the modeling was originally done. However, a formal, general analysis method has been developed [15], [7] by which the dc and small-signal transfer functions can be obtained directly without the use of an equivalent circuit at all, although such a circuit model can be extracted from an intermediate step.

This general analysis method is state-space averaging. Its essence lies in the fact that any pwm converter is a special kind of nonlinear system, one which is switched sequentially among two or more linear circuits according to one or more duty ratios. Furthermore, the "inputs" or control signals include not only independent voltages and currents, but also the duty ratios.

The basic buck, boost, flyback, and derived converters (such as the forward, push-pull, half- and full-bridge) are two-switched network converters when operated in continuous inductor current mode, in that the system is switched back and forth between two linear systems under the control of the duty ratio. The same converters operated in discontinuous inductor current mode [8], are threeswitched network systems, since the switching can be represented by a single-pole three-position switch. Only one of the three intervals is under independent control; the ratio between the remaining two intervals (defined by the inductor current falling to zero) is not an independent variable. However, there are other, more elaborate pwm converters that are multiple-switched networks in which all the duty ratios are independent control inputs.

Although state-space averaging accommodates all these cases, only the simplest, that applicable to two-switched networks in ccm, will be summarized here. The name itself is almost self-explanatory.

In each of the two positions of the switch, the system is linear and state-space equations can be written in the usual way. The state variables are the inductance currents and capacitance voltages, and a state-space equation is a firstorder differential equation. In matrix notation, the two sets of state-space equations are

$$
\begin{aligned}
& \dot{x}=A_{1} x+B_{1} u \\
& \dot{x}=A_{2} x+B_{2} u
\end{aligned}
$$

where $\boldsymbol{x}$ is the vector of state variables, $\boldsymbol{u}$ is the vector of independent sources (the line voltage, for example), and $A_{1}$, $\boldsymbol{B}_{1}$, and $\boldsymbol{A}_{2}, \boldsymbol{B}_{2}$ are respective system matrices in each of the two switched networks.

The key concept in state-space averaging is the replacement of the two sets of state-space equations by a single equivalent set

$$
\dot{x}=A x+B u
$$

in which the equivalent matrices $A$ and $B$ are weighted averages of the actual matrices that alternately describe the switched system. For a duty ratio $d$, the system spends a fraction $d$ of the switching period described by $A_{1}$ and $B_{1}$, and the remaining fraction $(1-d)$ described by $A_{2}$ and $B_{2}$. Hence [15], [7], the equivalent matrices are defined by

$$
\begin{aligned}
& \boldsymbol{A} \equiv d \boldsymbol{A}_{1}+(1-d) \boldsymbol{A}_{2} \\
& \boldsymbol{B} \equiv d \boldsymbol{B}_{1}+(1-d) \boldsymbol{B}_{2} .
\end{aligned}
$$

A single equivalent network can be found that is characterized by the matrices $\boldsymbol{A}$ and $\boldsymbol{B}$.

With the switched network now described by the equivalent matrices $\boldsymbol{A}$ and $\boldsymbol{B}$, the solution of (3) now proceeds in the usual manner. The steady-state solution, with dc values indicated by capital letters, is obtained by setting $\dot{x}=0$ :

$$
\boldsymbol{X}=-\boldsymbol{A}^{-1} \boldsymbol{B} U \text {. }
$$

The ac small-signal solution is found by the substitution $a$ $=\boldsymbol{U}+\boldsymbol{a}, \boldsymbol{x}=\boldsymbol{X}+\boldsymbol{x}$ into (3), in the usual way. However, the second key step peculiar to the switching converter application now arises, which involves recognition that the duty ratio $d$ is also modulated and constitutes an input; hence, the substitution $d=D+\hat{d}$ is also made in (3). Since this makes (3) nonlinear, the small-signal restriction becomes necessary in order that the equation may be linearized by omission of terms in the products of ac quantities. When this is done, and the dc terms are subtracted out, the resulting state-space average equation for the small-signal ac quantities is

$$
\hat{x}=\boldsymbol{A} \hat{x}+B \boldsymbol{B}+\boldsymbol{B}_{d} \hat{d}
$$


where

$$
\boldsymbol{B}_{d}\left(\boldsymbol{A}_{1}-\boldsymbol{A}_{2}\right) \boldsymbol{X}+\left(\boldsymbol{B}_{1}-\boldsymbol{B}_{2}\right) \boldsymbol{U}
$$

By Laplace transformation, the solution of (7) can be written

$$
\hat{\boldsymbol{x}}(s)=(s \boldsymbol{I}-\boldsymbol{A})^{-1}\left[\boldsymbol{B} \hat{\boldsymbol{u}}(s)+\boldsymbol{B}_{d} \hat{d}(s)\right]
$$

where $I$ is the identity matrix.

It often happens that the output signal required for some transfer function is not one of the state variables, but some combination of them and, in general, some direct function of the inputs, including the duty ratio. For example, if a converter output capacitor equivalent series resistance is accounted for, the converter output voltage is a function of both the capacitance voltage and inductance current state variables. In such cases, an output vector $y$ may be expressed for each of the two switched networks as

$$
\begin{aligned}
& y=C_{1} x+D_{1} u \\
& y=C_{2} x+D_{2} u
\end{aligned}
$$

and weighted averaging of the matrices is done in the same way as for $\boldsymbol{A}$ and $\boldsymbol{B}$. The same steps then lead to the results

$$
\begin{aligned}
Y & =C X+D U \\
\hat{y}(s) & =C \hat{x}(s)+D \hat{u}(s)+D_{d} \hat{d}(s)
\end{aligned}
$$

where the matrix coefficients are defined by (4), (5), and (8) with $\boldsymbol{C}, \boldsymbol{D}$, and $\boldsymbol{D}_{d}$ substituted for $\boldsymbol{A}, \boldsymbol{B}$, and $\boldsymbol{B}_{d}$, respectively.

Equations (12) and (13) provide, in closed form, the dc conditions and the small-signal frequency response of any pwm switching converter. The extension to more than two switched network systems is straightforward.

One of the uses of the results is that a single equivalent network can be found that is characterized by the averaged equations, which is the generalization of the circuit-oriented approach to modeling that was taken in the earlier sections. In particular, the averaging step corresponds to replacement of the switch by the conversion transformer, and the linearization step requiring the small-signal restriction corresponds to replacement of the conversion transformer varying ratio by a fixed-ratio transformer and the modulation generators. Note that the matrix coefficients $\boldsymbol{B}_{d}$ and $D_{d}$ of $\hat{d}$ are functions of operating point, as are the coefficients of the modulation generators in the circuit model.

The equivalent circuit models can be used either for analytic prediction of transfer functions, or can be presented as inputs to computer circuit analysis programs. If the program is capable of handling nonlinearities, the modulation of the conversion transformer can be accommodated directly without separation into the modulation generators [16].

Identification of an equivalent circuit model is, however, not necessary; the analytic results can be used directly to solve for any transfer function either numerically [17] or algebraically.

The state-space average modeling method is an almost ideal compromise between accuracy and simplicity. Both the equivalent circuit format, and the analytic results embodied in (12) and (13), are in forms familiar to electronic circuit engineers, and are therefore easily accessible. The principal limitation is that the results break down for modulation frequencies approaching the switching frequency, and indeed half the switching frequency is also a special case not fully accounted for in the results. However, as dis- cussed earlier, this is exactly the frequency range over which the results are needed, and extension of the range of validity requires rapidly increasing complexity of the model. Specifically, as discussed in [7], the inherent constraint required for the averaging step to be valid is that the corner frequency of the converter filter must be much lower than the switching frequency, by at least an order of magnitude. This condition is normally met in pwm converters, but resonant converters belong to a different class for which this condition is not met. State-space averaging is therefore not applicable, at least not directly, to resonant converters, and their models are not discussed in this paper.

\section{Conclusions}

Signal processing systems achieve desired functions with use of active devices, resistors, and capacitors, but inductors are avoided because of their incompatibility with integrated circuit fabrication techniques. Power processing systems, on the other hand, must avoid resistors in the interests of efficiency, and can incorporate only switches, capacitors, and magnetic structures.

A power processing system, such as a switched-mode dcto-dc regulated power supply, is also a signal processing system in that the regulator feedback loop must be characterized by appropriate transfer functions, which include those of the switched-mode modulator and power stage. Since the power stage is nonlinear, one seeks a suitable model from which the transfer functions can be obtained. As discussed in Section III, a suitable model is a small-signal ac model whose element values depend upon the large-signal dc operating point, but are otherwise constant.

Canonical models, of a given configuration but which have different element values for different converters and operating conditions, are developed qualitatively in Section IV. Fig. 5 shows the model for duty ratio programmed, pulse-width-modulated dc-to-dc converters operated in the continuous inductor current mode, in which the switching devices function as a two-position, single-pole switch. The model contains the three essential features of any such converter: the basic dc-to-dc conversion property, represented by the conversion transformer symbol which defines response down to dc; the effective low-pass filter property; and the adjustability property. Actually, adjustability is attained by modulation of the duty ratio $D$, of which the conversion ratio $M$ is a function; however, the model is linearized by representation of the adjustability property by separate voltage and current modulation generators. A duty ratio programmed converter is one whose duty ratio drive is a unique function of the control voltage, as delivered by a modulator that consists of a comparator driven by the control voltage and a fixed clock ramp, illustrated in Fig. 3.

Fig. 6 shows the canonical model for the same duty ratio programmed modulator as in Fig. 3, but with a power stage operated in the discontinuous inductor current mode, in which the switching devices function as a three-position single-pole switch. The same canonical model of Fig. 6 also applies to a current-programmed power stage, in which a local, or minor, current feedback loop is closed around the modulator-power-stage subsystem of Fig. 3, as illustrated in Fig. 7. The same $y$-parameter canonical model of Fig. 6 is appropriate for both modes of operation, because in both the output current follows the control voltage $\hat{v}_{c}$ and is therefore modeled by a voltage-controlled current gener- 
ator $y_{2 c} \hat{v}_{c}$. However, again in both modes of operation, the output current follows the control voltage only poorly, and the resistive component of the output admittance $y_{22}$ is not negligibly large, but comparable to the load resistance. Strictly speaking, neither mode of operation deserves the title "current-programmed," but this is the name conventionally adopted to describe the configuration of Fig. 7 in which an explicit attempt is made to achieve that result.

Comparison of the transfer functions as predicted by the canonical models for the three modes of operation is described in Section V, and summarized in Table 1. The current-programmed mode has dynamic-response properties in between those of the other two, having the major advantages of both, namely dominant pole response in the continuous inductor current mode, but with the disadvantage of a right half-plane zero in the boost and flyback type converters. Dominant-pole response (low $Q$, overdamped) is far preferable to the complex-pole (high $Q$, underdamped) response of the duty ratio programmed continuous inductor current mode since the damping is lossless, which considerably eases the efficiency versus dynamic response design tradeoff. This advantage, together with the simplicity with which switch current limit and paralleling of power stages can be achieved, makes current-programming the preferred mode of operation.

State-space averaging is the formal process of which the canonical equivalent circuit models are one form of the results. The method is summarized in Section VI. There are two key steps. One is the averaging step itself, in which the state-space equations describing the two or more switched networks are averaged according to the fractions of the switching period that the system resides in each configuration. The validity depends upon the corner frequency of the power stage filter being much lower than the switching frequency. The second key step accommodates the switch duty ratios as control inputs, subject to the small-signal limitation. Other useful forms of the results are analytic expressions for frequency responses, and numerical outputs of individual transfer functions [18].

Many authors have discussed the state-space averaging method [19]-[21], its limitations, and have made extensions to higher frequencies [22], larger signals [23], and to dc-toac converters [24]. Its major constraint, however, is that it does not apply to resonant converters, which constitute an important class for practical applications.

State-space averaging is not the only analysis method for pwm switching converters. Some alternative approaches are discussed in [25]-[30]. Simulation and computer-aided methods have also been employed [31]-[33].

REFERENCES

[1] R. D. Middlebrook and S. Ćuk, Advances in Switched-Mode Power Conversion, vols. I, II, and III. Pasadena, CA: TESLAco, 1983.

[2] S. Ćuk, "Integrated magnetics versus conventional power filtering," in Proc. INTELEC 87 Conf., pp. 61-72, 1987.

[3] F. Barzegar, S. Ćuk, and R. D. Middlebrook, "Using small computers to model and measure magnitude and phase of regulator transfer functions and loop gain," in Proc. Powercon 8 Conf., pp. H1.1-H1.28, 1981.

[4] G. W. Wester and R. D. Middlebrook, "Low-frequency characterization of switched dc-to-dc converters," in Proc. IEEE Power Electronics Specialists Conf., pp. 9-20, 1972; also IEEE Trans. Aerosp. Electron. Syst., vol. AES-9, pp. 376-385, May 1973.

[5] R. D. Middlebrook and S. Ćuk, "Modeling and analysis meth- ods for dc-to-dc switching converters," in Proc. IEEE /nt. Semiconductor Power Converter Conf., pp. 90-111, 1977.

[6] R. D. Middlebrook, "Topics in multiple-loop regulators and current-mode programming," in Proc. IEEE Power Electronics Specialists Conf., pp. 716-732, 1985.

[7] R. D. Middlebrook and S. Ćuk, "A general unified approach to modeling switching converter power stages," in Proc. IEEE Power Electronics Specialists Conf., pp. 18-34, 1976; also Int. J. Electron., vol. 42, no. 6, pp. 521-550, June 1977.

[8] S. Cuk and R. D. Middlebrook, "A general unified approach to modeling switching dc-to-dc converters in discontinuous conduction mode," in Proc. IEEE Power Electronics Specialists Conf., pp. 36-57, 1977.

[9] C. W. Deisch, "Simple switching control method changes power converter into a current source," in Proc. IEEE Power Electronics Specialists Conf., pp. 300-306, 1978.

[10] R. D. Middlebrook, "Topics in multiple-loop regulators and current-mode programming," IEEE Trans. Power Electron., vol. PE-2, pp. 109-124, Apr. 1987.

[11] S.-P. Hsu, A. Brown, L. Rensink, and R. D. Middlebrook, "Modeling and analysis of switching dc-to-dc converters in constant-frequency current-programmed mode," in Proc. IEFE Power Electronics Specialists Conf., pp. 284-301, 1979.

[12] R.D. Middlebrook, "Design techniques for preventing inputfilter oscillations in switched-mode regulators," in Proc. Powercon 5 Conf., pp. A3.1-A3.16, 1978.

[13] _-, "Design considerations and noise reduction in switching converters," Powerconversion Int. Mag., vol. 9, no. 8, pp. 20-30, Sept. 1983.

[14] - "Input filter considerations in design and application of switching regulators," in Proc. IEEE Industry Applications Society Annual Meeting, pp. 366-382, 1976.

[15] R. W. Brockett and J. R. Wood, "Electrical networks containing controlled switches," in "Applications of Lie group theory \& nonlinear network problems," supplement to IEEE Int Symp. on Circuit Theory, (San Francisco, CA), pp. 1-11, Apr. 1974.

[16] V.G. Bello, "Computer-modeling the pulse width modulated (PWM) converter," in Proc. Powercon 7 Conf., pp. G3.1-G3.11, 1980 .

[17] S. Ćuk, B. Lau, R. Gupta, and D. Borkovic, "Computer aided design of switching converter frequency response," in Proc. PowerCAD '87 Conf., pp. 106-121, 1987.

[18] SCAMP: Switching Converter Analysis and Measurement Program, Power Electronics Group, California Institute of Technology.

[19] G. Verghese and U. Mukherji, "Extended averaging and control procedures," in Proc. IEEE Power Electronics Specialists Conf., pp. 329-336, 1981.

[20] D. J. Shortt and F. C. Lee, "Extensions of the discrete-average models for converter power stages," in Proc. IEEE Power Electronics Specialists Conf., pp. 23-37, 1983.

[21] R. J. Dirkman, "Generalized state-space averaging," in Proc. IEEE Power Electronics Specialists Conf., pp. 283-294, 1983.

[22] A. R. Brown and R. D. Middlebrook "Sampled-data modeling of switching regulators," in Proc. IEEE Power Electronics Specialists Conf., pp. 349-369, 1981.

[23] R. W. Erickson, S. Ćuk, and R. D. Middlebrook, "Large-signal modeling and analysis of switching regulators," in Proc. IEEE Power Electronics Specialists Conf., pp. 240-250, 1982.

[24] K. D. T. Ngo, "Low frequency characterization of PWM converters," IEEE Trans. Power Electron., vol. PE-1, pp. 223-230, Oct. 1986.

[25] A. Capel, J. G. Ferrante, and R. Prajoux, "Dynamic behaviour and Z-transform stability analysis of DC/DC regulators with a nonlinear PWM controlled loop." in Proc. IEEE Power Electronics Specialists Conf., pp. 149-157, 1973.

[26] R. P. Iwens, Y. Yu, and J. E. Triner, "Time domain modeling and stability analysis of an integral pulse frequency modulated DC to DC power converter," in Proc. IEEE Power Electronics Specialists Conf., pp. 80-90, 1975.

[27] A. Capel, J. G. Ferrante, and R. Prajoux, "State variable stability analysis of multi-loop PWM controlled regulators in light and heavy mode," in PrOc. IEEE Power Electronics Specialists Conf., pp. 91-102, 1975.

[28] F. C. Lee, Y. Yu, and J. E. Triner, "Modeling of switching regulator power stages with and without zero-inductor-current 
dwell time," in Proc. IEEE Power Electronics Specialists Conf., pp. $62-72,1976$.

[29] R. P. Iwens, F. C. Lee, and J. E. Triner, "Discrete time domain modeling and analysis of dc-dc converters with continuous and discontinuous inductor current," presented at the Proc. 2nd IFAC Symp. of Control in Power Electronics and Electrical Drives, Dusseldorf, West Germany, Oct. 1977.

[30] G. Verghese, M. E. Elbuluk, and J. G. Kassakian, "A general approach to sampled-data modeling for power electronic circuits," IEEE Trans. Power Electron., vol. PE-1, pp. 76-89, Apr. 1986.

[31] A. K. Ohri, H. A. Owen, Jr., T. G. Wilson, and G. E. Rodrigues, "Digital computer simulation of inductor-energy-storage converters with closed-loop regulators," in Proc. Spacecraft Power Conditioning Electronics Seminar, (Frascati, Italy), May 1974; ESRO Pub. SP-103, pp. 245-261.

[32] H. A. Owen, A. Capel, and I. G. Ferrante, in Proc. IEEE Power Electronics Specialists Conf., pp. 45-55, 1976.

[33] F. C. Y. Lee, R. P. Iwens, Y. Yu, and J. E. Triner, "Generalized computer-aided discrete-time modeling and analysis of dc-dc converters," IEEE Trans. Indust. Electron. Contr. Instrum., vol. IECI-26, pp. 58-69, May 1979.

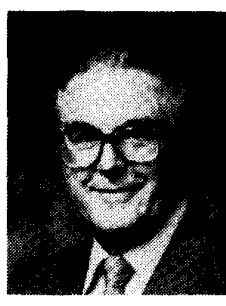

R. D. Middlebrook (Fellow, IEEE) received the Ph.D. degree in electrical engineering from Stanford University, Stanford, CA, in 1955.

$\mathrm{He}$ is Professor of Electrical Engineering at the California Institute of Technology, Pasadena, CA. His publications include numerous papers, a book on solid-state device theory, and another on differential amplifiers. His research interests, formerly in solid-state device modeling, are now in circuits and systems, and particularly in power processing electronics, in which he is well known as author lecturer, and consultant. He is especially interested in design-oriented circuit analysis and measurement techniques which he teaches at Caltech, and has conducted short courses on his methods in both Europe and the United States. Among his outside interests is Formula race car driving.

Dr. Middlebrook is the recipient of the 1982 IEEE William E. Newell Power Electronics Award for Outstanding Achievement in Power Electronics, and a 1982 Award for Excellence in Teaching, presented by the Board of Directors of the Associated Students of Caltech. 